\title{
Theoretical approaches for determining machining conditions affecting a machined surface topography in filleted end milling
}

\author{
Tsutomu Sekine* (iD \\ Department of Systems Design Engineering, Faculty of Science and Technology, Seikei University, Tokyo 180-8633, Japan
}

Received: 10 April 2021 / Accepted: 3 October 2021

\begin{abstract}
This study demonstrates theoretical approaches useful for practical determination of machining conditions affecting machined surface topography in filleted end milling. Tool orientation is investigated in particular. There are dominant processing parameters' optimizations from various perspectives, whereas a few comprehensive strategies have been proposed to determine machining conditions in filleted end milling. It is also practically scarce to discover the optimization strategy taking path interval determination as the theoretical fountainhead. In this study, theoretical approaches were described to determine machining conditions affecting machined surface topography in filleted end milling. After geometrical description was arranged to model multiaxis filleted end milling, multi-layer approach and the other computable parameters were proposed to obtain decent surface topography generated in filleted end milling. The analytical example focusing on tool orientation was provided with discussion. As a result, some characteristics of theoretical approaches were revealed with the visual evidences. Finally, optimal tool orientation will be arranged based on the findings.
\end{abstract}

Keywords: Machined surface / surface roughness optimization / path interval / multi-axis CNC machining / filleted end mill

\section{Introduction}

Surface topography is one of the vital factors in product features. The manufacturing technologies are being continuously required to create a value-added surface in many industries such as automobile, aerospace, and electronic device. Against the background of industrial demands, a lot of contributions have been made from various perspectives [1-4]. Among them, a machined surface in milling is attracting persistent attention from industrial society and researchers [5-11]. The major factors affecting a machined surface has been gradually revealed and considered mostly as cutting parameters, thermal parameters, dynamic parameters, machine tool parameters, tool properties, and workpiece properties [12]. As the technical findings, controlling surface topography in milling enables us to add the surface to a functional property $[13,14]$. It is also well known that optimizing a path interval in tool path generation can improve not only surface topography but also a balance between cost and product quality in milling $[15,16]$. The methodologies to determine a single optimum point have been mostly developed based on an algebraic equation derived from geometrical analysis of milling process.

\footnotetext{
* e-mail: ts_s@outlook.com
}

There exist two path intervals on a surface machined by milling. The one is a path interval along the feed direction of a tool, and the other is a path interval along the cross-feed direction of a tool. Path interval determination have mostly focused on the latter $[17,18]$. Scallop height acts as a dominant factor in the prediction of path interval. Although there are various kinds of tools for milling in these days, typical tool tip geometries have been studied so far in path interval determination with various machining situations [19-23]. Among them, multi-axis flat and filleted end milling requires the elaborate treatments according to a machining situation. There are four general classifications of the situations in multi-axis filleted end milling [24]. The derivation of geometrical relationships tends to be unachievable with the two-dimensional expression for the cutting edge geometry, whereas that of three-dimensional (3D) expression entails mathematical complexities according to intersection problems of 3D geometries [25]. A novel procedure to overcome the complexities was reported with respect to an accurate prediction of path interval in filleted end milling with a tool inclination [26]. The results represented that introducing a reference point was important to estimate a path interval correctly in the 3D consideration.

It is crucial to optimize a milled surface topography in high level through selecting suitable machining parameters in milling. There are a variety of methodologies aimed at a 
point-based optimization of machining parameters in milling [27-31]. Cutting force is one of the high-interest topics to enhance finish surface quality and to avoid tool failure. Lazoglu et al. proposed a feedrate scheduling technique based on their force model [32]. Habibi et al. also reported a computationally-inexpensive approach minimizing flute engagement to adjust tool orientation for optimizing surface errors in five axis ball end milling [33]. Moreover, cutter/workpiece engagement is an important factor to figure out suitable processing parameters. Zhang et al. investigated an optimization of tool orientation in 5axis ball-end milling through a model of cutter/workpiece engagement [34]. Chip thickness is theoretically modeled to estimate the other machining factors. Lotfi et al. gave a model of instantaneous undeformed chip thickness [35]. Residual stress in machined surface is also a measure to optimize processing parameters; in addition, Masmiati and Sarhan revealed an effect of cutting parameters on residual stress in machined surfaces [36]. Although there are dominant processing parameters' optimizations from various perspectives, a few comprehensive strategies have been proposed to determine machining conditions in filleted end milling. The one based on path interval determination has been scarcely reported so far in practice.

The purpose of this study is to demonstrate theoretical approaches useful for practical determination of machining conditions affecting machined surface topography in filleted end milling. Tool orientation is intensively investigated in particular. The remainder of this paper is organized as follows. Section 2 describes geometrical description of multi-axis filleted end milling. Then, multi-layer approach will be explained to determine a suitable path interval in Section 3. The other theoretical approaches for several dominant processing parameters will also be given in Section 4. The demonstration and discussion are made in Section 5. Finally, conclusion and future work are presented in Section 6.

\section{Geometrical description of filleted end milling}

Several coordinate systems is firstly provided to express a machining state of filleted end milling. Henceforth, an axis of a coordinate system is invariably denoted by a normalized vector. This study introduces three coordinate systems as shown in Figure 1. These right-handed coordinate systems are labelled as G, M, and T. G coordinate system comprises $\mathbf{X}, \mathbf{Y}, \mathbf{Z}$ axes, whose components are $(1,0,0),(0,1,0)$, and $(0,0,1)$, respectively. $\mathrm{M}$ coordinate system is also defined based on a surface to be machined. $\mathbf{M Z}$ is a unit surface normal on a workpiece. $\mathbf{M Y}$ is orthogonal to $\mathbf{M X}$, and it is oriented to the scanning direction of a tool; moreover, $\mathbf{M Y}$ can be obtained from the cross product of $\mathbf{M X}$ and $\mathbf{M Z}$.

Two sections are instantaneously set to consider the other coordinate system and inclination angles in filleted end milling. The one is a section based on the scanning direction of a tool (i.e., MZ-MX plane), while the other is a section based on the cross-feed direction perpendicular to the scanning direction (i.e., $\mathbf{M Z}-\mathbf{M Y}$ plane). Let these planes be scanning

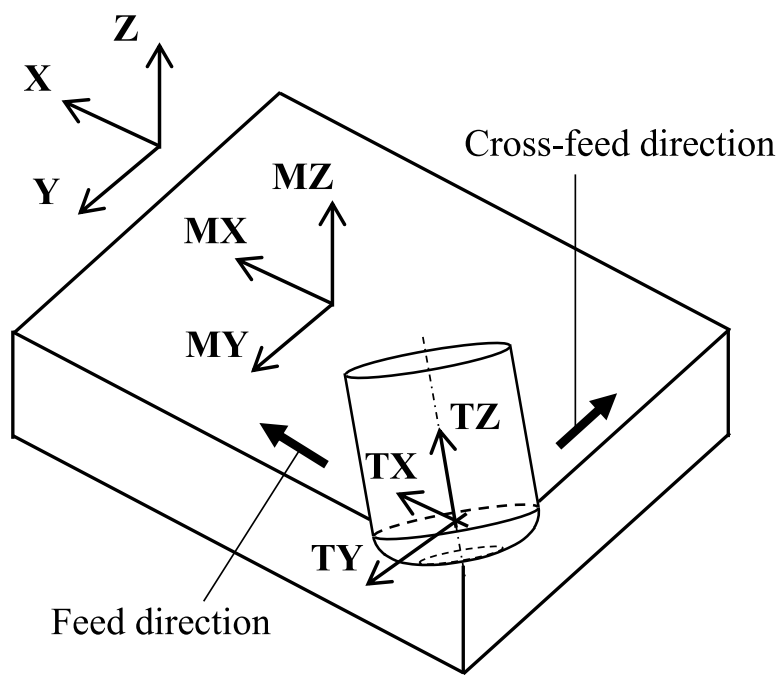

Fig. 1. Three coordinate systems in filleted end milling.

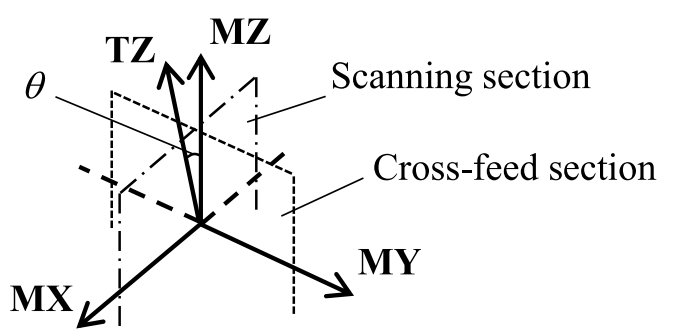

Fig. 2. Two instantaneous sections and tool inclination angle $\theta$ between $\mathrm{MZ}$ and $\mathrm{TZ}$.

section and cross-feed section, respectively. $\mathrm{T}$ coordinate system can be expressed using the two sections. The position of $\mathbf{T Z}$ corresponds to that of the rotational axis of a tool, and the direction is oriented to the shank side of tool. As illustrated in Figure 2, tool inclination angle $\theta$ denotes the angle between $\mathbf{M Z}$ and $\mathbf{T Z}$. TX is geometrically calculated as a vector perpendicular to $\mathbf{T Z}$. The direction depends on the directional relationship between TZ and MZ. Specifically, the angle between $\mathbf{T X}$ and $\mathbf{M X}-\mathbf{M Y}$ plane is inevitably set to be $\theta$. Then, TY can be obtained from the cross product of TX and TZ.

The angles $\theta_{s}$ and $\theta_{c}$ are additionally introduced to derive $\theta$. As shown in Figure $3, \theta_{s}$ is the inclination angle on scanning section, designating the angle between $\mathbf{M Z}$ and the orthogonal projection of $\mathbf{T Z}$ onto scanning section. Likewise, $\theta_{c}$ is the inclination angle on cross-feed section, indicating the angle between $\mathbf{M Z}$ and the orthogonal projection of $\mathbf{T Z}$ onto cross-feed section. Counter clockwise rotation is the positive rotational direction of $\theta_{s}$ and $\theta_{c}$.

Given that M coordinate system coincides completely with $\mathrm{G}$ coordinate system for simple modeling of filleted end milling, $\theta_{s}$ and $\theta_{c}$ can be calculated as follows:

$$
\theta_{s}=\cos ^{-1}\left(\frac{T Z_{z}}{\sqrt{T Z_{y}^{2}+T Z_{z}^{2}}}\right)
$$



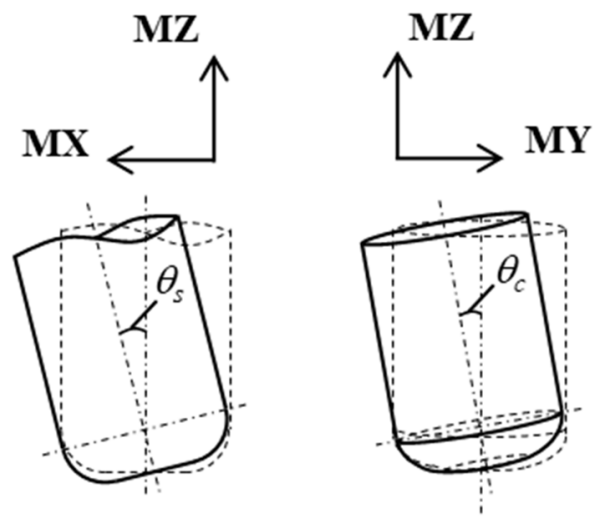

Fig. 3. Inclination angles $\theta_{s}$ and $\theta_{c}$.

$$
\theta_{c}=\cos ^{-1}\left(\frac{T Z_{z}}{\sqrt{T Z_{x}^{2}+T Z_{z}^{2}}}\right)
$$

where $T Z_{x}, T Z_{y}$, and $T Z_{z}$ are three components of $\mathbf{T Z}$ in each axis of $\mathrm{G}$ coordinate system. Since $\mathbf{T Z}$ is given as a normalized vector,

$$
T Z_{x}^{2}+T Z_{y}^{2}+T Z_{z}^{2}=1
$$

Hence, equations (1) and (2) can lead to the following components of $\mathbf{T Z}$ :

$$
\begin{gathered}
T Z_{x}=T Z_{z} \tan \theta_{s} \\
T Z_{y}=-T Z_{z} \tan \theta_{c}
\end{gathered}
$$

Substituting equations (4) and (5) to equation (3) provides the following formula:

$$
T Z_{z}=\sqrt{\frac{1}{\tan ^{2} \theta_{c}+\tan ^{2} \theta_{s}+1}}
$$

Accordingly, the angle $\theta$ can be offered as an inverse trigonometric function with $T Z_{z}$ :

$$
\theta=\cos ^{-1}\left(\frac{T Z_{z}}{\sqrt{T Z_{x}^{2}+T Z_{y}^{2}+T Z_{z}^{2}}}\right)=\cos ^{-1}\left(T Z_{z}\right)
$$

Figure 4 illustrates the positional relationship of two cutting edges in filleted end mill. Although there exist various kinds of cutting edge geometry, this study focuses on a filleted end mill with two flutes and without a helix angle of cutting edges. With reference to Figure 4, a cutting point on a cutting edge in $\mathrm{T}$ coordinate system $\mathbf{T P e}$ can be calculated as follows: when $d_{a e} \geq R-R_{c r}$,

$$
\begin{aligned}
& T P e_{x}=\left(\left(R-R_{c r}\right)+R_{c r} \sin \psi\right) \sin (\xi+\zeta), \\
& T P e_{y}=\left(\left(R-R_{c r}\right)+R_{c r} \sin \psi\right) \cos (\xi+\zeta), \\
& T P e_{z}=-R_{c r} \cos \psi
\end{aligned}
$$

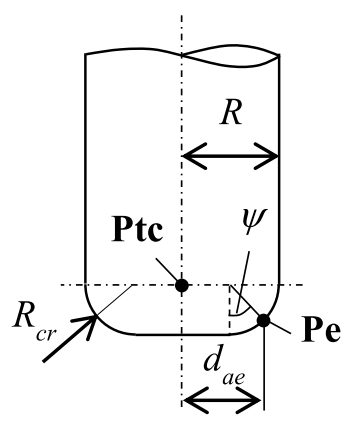

(a) Typical tool profile

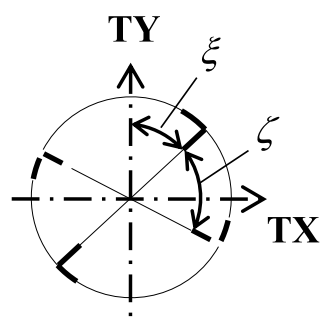

(b) Cutting edges' position in TX - TY plane

Fig. 4. Typical tool profile and cutting edges' position.

and when $d_{a e}<R-R_{c r}$,

$$
\begin{aligned}
& T P e_{x}=d_{a e} \sin (\xi+\zeta), \\
& T P e_{y}=d_{a e} \cos (\xi+\zeta), \\
& T P e_{z}=-R_{c r}
\end{aligned}
$$

It should be careful that clockwise rotation is the positive rotational direction of $\xi$ and $\zeta$. Common end mills have a number of cutting edges, so that $\xi$ depends on the number of cutting edges $n_{c e}$. In the case that the angular position of a cutting edge overlaps TX axis, $\xi$ can be derived as a simple form:

$$
\xi=(m-1) \frac{2 \pi}{n_{c e}} \quad\left(1 \leq m \leq n_{c e}\right)
$$

where $m$ is an assigned number of a cutting edge. From the above explanation, a cutting point on a cutting edge $\mathbf{P e}$ can be calculated through the following formula:

$$
\mathbf{P e}=\mathbf{R}_{\mathbf{T}} \mathbf{T P e}+\mathbf{P t c}
$$

where $\mathbf{R}_{\mathbf{T}}$ is coordinate transformation matrix, providing a transformation from $\mathrm{T}$ to $\mathrm{O}$ coordinate system; moreover, Ptc is an instantaneous tool center shown in Figure 4a, depending on a tool path up until a present position. The geometrical description explained in this section assumes a filleted end mill without a helix angle of cutting edges, while it can effortlessly apply to the common, commercial ones through dividing a tool into small disk elements. 


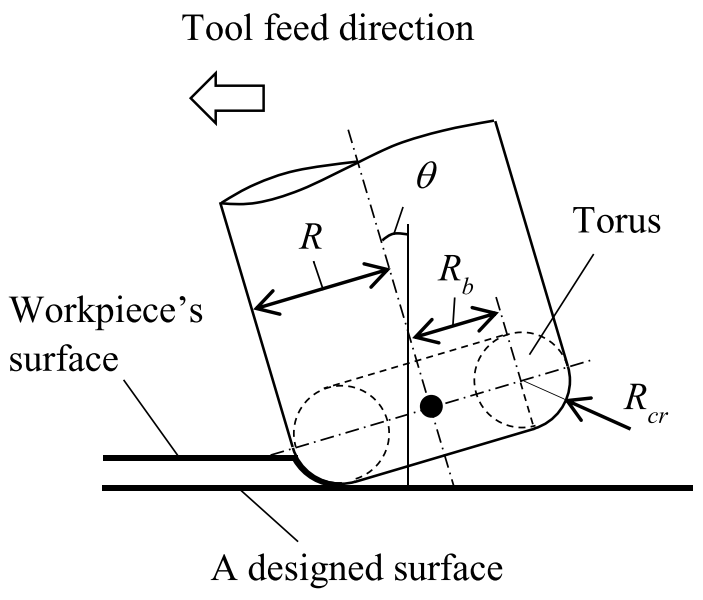

Fig. 5. An assigned torus and several radii.

\section{Multi-layer approach}

This section describes a novel procedure for theoretical estimation of machined surface topography. Multi-layer approach is introduced as a careful, effectual selection procedure with path interval determination. There are two path intervals associated with a machined surface topography after milling. The one is a path interval in cross-feed direction, and the other is a path interval in feed direction. Let each path interval be path interval $L_{c}$ and feed interval $L_{f}$, respectively. From these definitions, scallop heights can also be given as scallop height $h_{c}$ and feed mark's height $h_{f}$, respectively.

Considering a variation of the tool orientation for determining a path interval $L_{c}$, a variety of the tool orientation derived from a tool geometry projected onto an instantaneous section can be classified into four cases according to $\theta_{s}$ and $\theta_{c}[24]$. The possible situations in $3 \mathrm{D}$ geometry can be considered thoroughly based on a tool inclination angle $\theta$. This section mainly deals with filleted end milling under the case that $\theta \neq 0$.

\subsection{Multi-layer concept derived from path interval determination}

Two path intervals commonly express a distance between adjacent tool centers in a tool trajectory. Although a path interval in cross-feed direction $L_{c}$ can be generally calculated as a point-to-point distance, this study focuses mainly on $L_{c} / 2$. This variable indicates a distance from a tool center to an expediential section located with a predetermined scallop height $h_{c}$. Figure 5 illustrates several radii, i.e. $R, R_{b}$, and $R_{c r}$, of filleted end mill with a tool inclination angle $\theta$. A torus is used to express machining states in the cutting edge geometry of filleted end mill.

A machining situation of cutting edge geometry is given in Figure 6, and a torus is used as a model of cutting edge. The coordinate systems in Figure 6 are identical to the ones in Figure 1. The direction of $\mathbf{Y T}$ axis is the same as that of $\mathbf{Y}$ axis, and tool feed direction coincides with the direction of $\mathbf{X}$ axis. There are several planes, i.e. a designed surface,

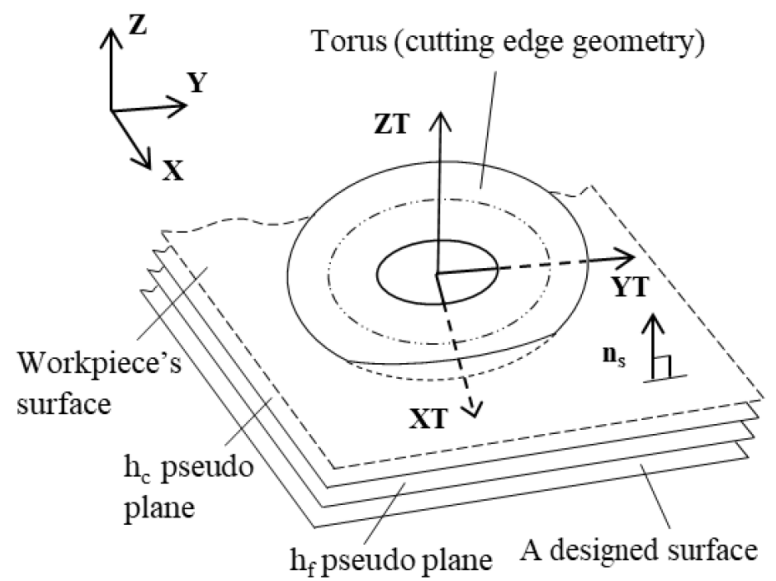

Fig. 6. An assigned torus and several radii.

workpiece's surface, and two pseudo planes regarding a scallop height $h_{c}$ and a feed mark's height $h_{f}$. The distance of two planes is situationally changeable in accordance with a variation of designed surface topography. $\mathbf{n}_{\mathbf{s}}$ is given as a surface normal in each surface and plane. The direction of $\mathbf{n}_{\mathbf{s}}$ changes depending on the orientation of reference surface or plane.

\subsection{Path interval determination in a cross-feed direction}

Figure 7 shows the pseudo-code of path interval determination in filleted end milling [26]. The algorithm focuses on $h_{c}$ pseudo plane as an exemplified explanation, whereas the computational process is similarly available for workpiece's surface. In the algorithm, a torus has inclination $\theta$ and contacts at a point on a designed surface. When a section as an exact circle is cut out from the torus, there exist three positional relationships between a torus section and $h_{c}$ pseudo plane. The one is an intersection between a torus section and $h_{c}$ pseudo plane, while there exists a positional relationship without an intersection. The other situation is a single contact point between a torus section and $h_{c}$ pseudo plane.

The notation $\mathbf{P}, \mathbf{t}$, and $\mathbf{u}$ indicate a positional vector in $3 \mathrm{D}$ space, a tangent vector at each torus section's center, and a directional vector. Each subscript of these vectors is mainly associated with the positional relationships between a torus section and $h_{c}$ pseudo plane. The subscript base is used for $\mathbf{P}_{\text {base }}$ and $\mathbf{t}_{\text {base }}$ which express vectors at torus section's center with a contact point between the torus and a designed surface. Moreover, the distance between $\mathbf{P}_{\text {base }}$ and a designed surface is completely equal to $R_{c r}$. The subscript ap is used to express an arbitrary position. $\gamma$ is an angular parameter for determining an arbitrary position on a circle with $R_{b}$. Rearranging the following formula can provide the initial value of $\gamma$ designated as $\gamma_{a p}$ in Figure 7:

$$
R_{b} \sin \theta-R_{b} \cos \gamma \sin \theta=0.5 h_{c}
$$

A search range in iterative calculation is introduced along a circle with $R_{b}$. $\mathbf{P}_{\text {ap }}$ is an arbitrary position on the 
Algorithm: Path interval determination

Input: $R, R_{c r}, \theta, h_{c}$

Output: $L_{c} / 2$

1: Set $\mathbf{X}, \mathbf{Y}, \mathbf{Z}, \mathbf{X T}, \mathbf{Y T}, \mathbf{Z T}, \mathbf{n}_{\mathbf{s}}, \mathbf{P}_{\text {base, }}$, and $\mathbf{t}_{\text {base }}$

2: Calculate $\gamma_{a p}$ using Eq. (1)

3: Calculate $\eta$ using Eq. (2)

4: Calculate $d_{s}$ using Eq. (3)

5: Set a search range $\mathbf{P}_{\text {start }}$ and $\mathbf{P}_{\text {end }}$

for the following iterative calculation

6: do

7: Update $\gamma$ according to $R_{c r}-d_{s}$

8: Update $\mathbf{P}_{\text {ap }}$ and $\mathbf{t}_{\mathbf{a p}}$

9: Calculate $\eta$ using Eq. (2)

10: Calculate $d_{s}$ using Eq. (3)

11: while $\left|R_{c r}-d_{s}\right| \geq \varepsilon$

12: $\mathbf{m}_{\text {scp }} \leftarrow \mathbf{t}_{\text {scp }} \times \mathbf{n}_{\text {s, }}, \mathbf{u}_{\text {scp }} \leftarrow \mathbf{t}_{\text {scp }} \times \mathbf{m}_{\text {scp }}$

13: $\mathbf{P}_{\mathrm{hp}} \leftarrow \mathbf{P}_{\mathrm{scp}}+R_{c r} \mathbf{u}_{\mathrm{scp}}$

14: $\gamma_{s c p} \leftarrow \gamma$

15: Calculate $\gamma$ using Y-axis component of $\mathbf{P}_{\text {scp }}$ to set a search range for the next iterative calculation

16: Let $\mathbf{P}_{\mathrm{hp}}[1 \ldots n]$ and $\gamma[1 \ldots n]$ be new arrays through dividing the search range from $\gamma$ to $\gamma_{s c p}$ into appropriate even numbers

17: $\gamma[1] \leftarrow \gamma, \gamma[n] \leftarrow \gamma_{s c p}$

18: Calculate $\mathbf{P}_{\mathbf{h p}}$ [1] and $\mathbf{P}_{\mathbf{h p}}[n]$

19: do

20: for $i=2$ to $n-1$

21: $\quad$ Calculate $\gamma[i]$ and $\mathbf{P}_{\mathbf{h p}}[i]$

22: end for

23: Find $\mathbf{P}_{\mathbf{h p}}[i]$ with the maximum of $\mathrm{Y}$-axis component

23: Rearrange $\mathbf{P}_{\mathbf{h p}}$ [1] and $\mathbf{P}_{\mathbf{h p}}[n]$ using the elements near $\mathbf{P}_{\mathbf{h p}}[i]$ calculated above, and update $\gamma[1]$ and $\gamma[n]$

24: Compute the difference between $\mathbf{P}_{\mathbf{h p}}[i]$ calculated above and the adjacent $\mathbf{P}_{\mathbf{h p}}[i]$ in $\mathrm{Y}$-axis component

25: while the difference $\geq \varepsilon$

26: $L_{c} / 2 \leftarrow \max \left\{\mathrm{Y}\right.$-axis component of $\mathbf{P}_{\mathbf{h p}}[i]: i=1$ to $\left.n\right\}$

Fig. 7. Computational algorithm for determining $L_{c} / 2[26]$.

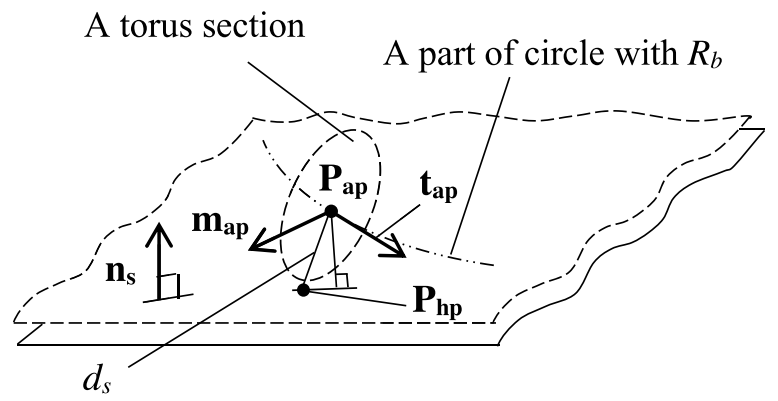

Fig. 8. Some variables associated with a torus section.

circle. $\mathbf{P}_{\text {start }}$ and $\mathbf{P}_{\text {end }}$ are temporarily provided as the starting and ending point of search range, respectively. $\mathbf{P}_{\text {start }}$ is a position vector when $\gamma=0$, while $\mathbf{P}_{\text {end }}$ is a position vector when $\gamma=0.5 \pi$. A position vector of $\mathbf{P}_{\text {ap }}$ can be easily derived through rotating $\mathbf{P}_{\text {base }}$ around $\mathbf{Z T}$ axis. Moreover, a tangent vector $\mathbf{t}_{\mathbf{a p}}$ can be also calculated using $\mathbf{t}_{\text {base }}$ in the same manner. Figure 8 illustrates some variables in a torus section at $\mathbf{P}_{\mathbf{a p}} . \mathbf{P}_{\mathbf{h p}}$ indicates a positional vector on $h_{c}$ pseudo plane. A direction cosine $\eta$ between $\mathbf{t}_{\mathbf{a p}}$ and $\mathrm{h}_{\mathrm{c}}$ pseudo plane can be obtained by the following equation:

$$
\eta=\mathbf{t}_{\mathrm{ap}} \cdot\left\{\left(\mathbf{n}_{\mathbf{h}} \times \mathbf{t}_{\mathrm{ap}}\right) \times \mathbf{n}_{\mathbf{h}}\right\}
$$

A distance $d_{s}$ between $\mathbf{P}_{\text {ap }}$ and $\mathbf{P}_{\mathbf{h p}}$ can be given as follows:

$$
d_{s}=\frac{\left(R_{c r}-h_{c}+R_{b} \sin \theta-R_{b} \cos \gamma \sin \theta\right)}{\eta}
$$

The subscript scp means a single contact point between a torus section and $\mathrm{h}$ pseudo plane. $\mathbf{P}_{\mathbf{s c p}}$ and $\mathbf{t}_{\mathbf{s c p}}$ are obtained through updating $\mathbf{P}_{\text {ap }}$ and $\mathbf{t}_{\mathbf{a p}}$ in iterative calculation, which is the first do-while statement in Figure 7. $\varepsilon$ is set as the convergence condition of iterative calculation. The second iterative calculation in Figure 7 can ascertain an intersection between a torus section and $h_{c}$ pseudo plane. Through finding out the farthest intersection from the tool center point, the algorithm can provide a suitable path interval $L_{c} / 2$.

\subsection{Path interval determination in a feed direction}

Feed interval $L_{f}$ and feed mark's height $h_{f}$ have been scarcely studied so far. The interval is closely associated with feed per tooth $f_{t}$. A tool moves along with $\mathbf{X}$ axis, and the direction of $\mathbf{T X}-\mathbf{T Z}$ plane includes a contact point between a torus and a designed surface. Here, let $\omega$ be the rotational angle between $\mathbf{X}$ axis and $\mathbf{T X}-\mathbf{T Z}$ plane. The rotation is about $\mathbf{Z}$ axis. Then, the following formula can be made to calculate a feed interval:

$$
L_{f}=f_{t} \cos \omega
$$


where $f_{t}$ is feed per tooth. Moreover, feed mark's height $h_{f}$ can be mathematically expressed in the same manner to path interval determination in ball end milling.

\section{Estimation of the other dominant processing parameters}

A milled surface topography can be directly and indirectly affected by the other dominant processing parameters, so that the effectual selection procedures for determining these parameters are also indispensable to obtain a decent topography. With the aim of optimal parameters' selection, this section provides the some theoretical procedures in filleted end milling. Note that the following calculations are unalterably given for one cutting edge during one rotation of a tool.

\subsection{Maximum frictional distance}

Frictional distance is a critical factor to predict tool wear at the cutting edge. It also has an influence on a machined surface feature. The following formula can provide the maximum length:

$$
s l_{f d}=\left(R_{c r} \sin \alpha_{f d}+R_{b}\right) \beta_{f d}
$$

In the above formula, $\alpha_{f d}$ is obtained from an arccosine calculation for a scalar product of $\mathbf{u}_{\mathbf{s c p}}$ and $-\mathbf{T Z}$. Moreover, a rotational angle around $\mathbf{T Z}$ axis $\beta_{f d}$ is utilized as the one between $\mathbf{T X}-\mathbf{T Z}$ plane and a center of torus section having a contact point with a workpiece's surface. Each angle can be extracted as partial results arising inevitably and functionally in the execution of computational algorithm.

\subsection{Maximum contact arc length}

Contact arc length is defined based on a cutting-related part in a cutting edge, so that it is also an important factor in considering tool surface damage and a machined surface feature. The maximum length can be expressed:

$$
s l_{c a l}=R_{c r}\left(\alpha_{c a l}+\alpha_{h f}\right)
$$

where $\alpha_{c a l}$ is easily identified from an angle calculation using $R_{c r}$ and the depth of cut $d_{d o c}$ which means a distance between a designed surface and workpiece's surface principally. In contrast, $\alpha_{h f}$ can be computed from an angle calculation based on a feed mark's height $h_{f}$. The execution of computational algorithm including a partial result arising inevitably and functionally can also offer the angle.

\subsection{Maximum cutting speed}

It is well known that cutting speed has actual impact on both tool surface damage and cutting force. The following expression can be made to estimate the maximum cutting speed:

$$
v_{c s}=2 \pi\left(R_{b}+R_{c r} \cos \left(\frac{\pi}{2}-\left(\theta+\alpha_{c s}\right)\right)\right) \frac{s}{1000}
$$

Table 1. The machining conditions in filleted end milling.

\begin{tabular}{ll}
\hline Tool radius, $R[\mathrm{~mm}]$ & 6.0 \\
Tool tip radius, $R_{c r}[\mathrm{~mm}]$ & 4.0 \\
The number of tooth, $n_{c e}$ & 2 \\
Dipth of cut, $d_{d o c}[\mathrm{~mm}]$ & 0.2 \\
Scallop height in cross-feed direction, $h_{c}[\mathrm{~mm}]$ & 0.001 \\
Tool rotational speed, $s\left[\mathrm{~min}^{-1}\right]$ & 1400 \\
Feed rate, $f[\mathrm{~mm} / \mathrm{min}]$ & 260 \\
\hline
\end{tabular}

where $s$ is a spindle speed in cutting process, and $\alpha_{c s}$ is easily identified using the depth of cut $d_{d o c}$. The execution of computational algorithm with partial functions can instantaneously compute the angle.

\subsection{Average uncut chip thickness}

Uncut chip thickness is one of the vital factors having great impact on cutting force. It is undoubted that uncut chip thickness affects tool surface damage in practice since cutting force directly depends on material removal rate in machining process. The following formula enables us to calculate the average value:

$$
t_{u c t}=f_{t} \sin \beta_{u c t} \sin \alpha_{u c t}
$$

where $\alpha_{u c t}$ indicates an angular parameter for determining an arbitrary position on a cutting edge. In addition, $\beta_{\text {uct }}$ designates an angle between $\mathbf{T X}$ axis and $\mathbf{G Y}-\mathbf{G Z}$ plane, and the rotation is about $\mathbf{T Z}$ axis. The above formula can estimate an uncut chip thickness at any position on a cutting edge. As a brief estimation, an average uncut chip thickness $t_{\text {ave }}$ was computed in a cutting edge's rotational position where the instantaneous cutting load and area were the largest in both measures, and three angles of $\alpha_{u c t}$ were considered for the calculation. The first angle was obtained based on the depth of cut $d_{d o c}$. The second angle was set to 5 deg. a priori. Finally, their median was also set to the third angle, and the average calculation was conducted with $t_{u c t}$ obtained using these angles.

\section{Demonstration and discussion}

This section describes a demonstration of the estimation approaches proposed above. Especially, we investigated influence of tool orientation on each dominant processing parameter in filleted end milling. The discussion will be given after visualizing characteristics of these parameters. The machining conditions used in the demonstration are shown in Table 1. The tool diameter of end mill was set on the basis of a commonly-used size, and the tool had two straight cutting edges. The unit of angle was set as deg. to aid an intuitive understanding.

The influence of tool orientation on $\theta$ is shown in Figure 9. In the figure, each curve for respective $\theta_{s}$ in graph legends represented increasing tendency with increasing $\theta_{c}$ 


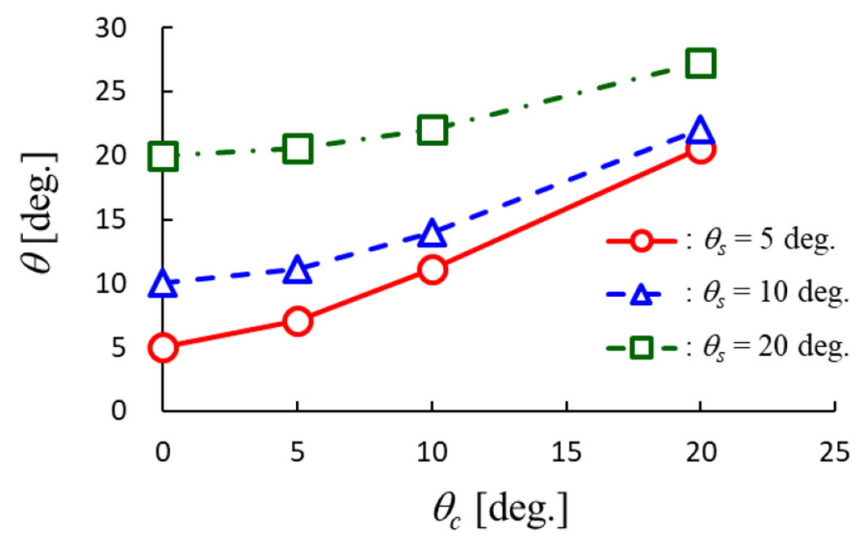

Fig. 9. Influence of $\theta_{s}$ and $\theta_{c}$ on $\theta$.

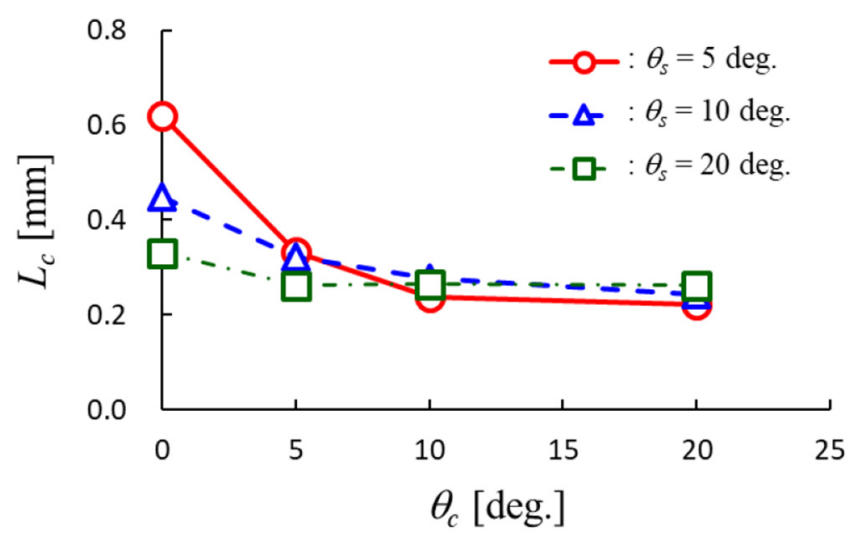

Fig. 10. Influence of $\theta_{s}$ and $\theta_{c}$ on $L_{c}$.

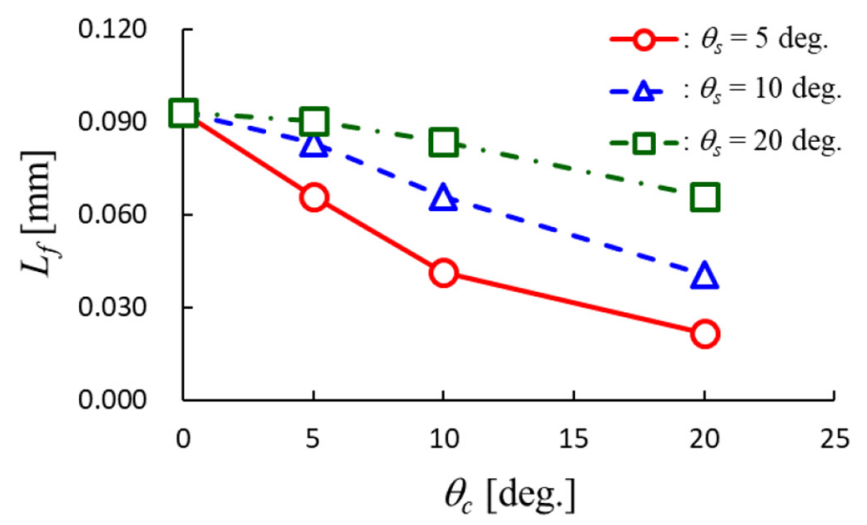

Fig. 11. Influence of $\theta_{s}$ and $\theta_{c}$ on $L_{f}$.

in any case. The increasing tendency gradually diminished with increasing $\theta_{s}$. Moreover, the differences between each curve became smaller with increasing $\theta_{c}$. The results indicated that the variation of $\theta$ depended largely on the larger one of two angles $\theta_{s}$ and $\theta_{c}$. As a numerical example, when $\theta_{s}=20 \mathrm{deg}$. and $\theta_{c}=5 \mathrm{deg}$., the value of $\theta$ is $20.52 \mathrm{deg}$. It was obvious that the effect of $\theta_{c}$ on the value of $\theta$ was extremely small.
The influence of tool orientation on $L_{c}$ is given in Figure 10. In the figure, each curve for respective $\theta_{s}$ in graph legends showed decreasing tendency with increasing $\theta_{c}$ in any case. The decreasing tendency gradually reduced with increasing $\theta_{s}$, and an asymptotical behaviour could be observed in any curve. Moreover, the differences between each curve became drastically smaller with increasing $\theta_{c}$. The results clearly expressed that the value of $L_{c}$ was independent of tool orientation in the case of $\theta$ having an angle more than $10 \mathrm{deg}$. To take a numerical example, when $\theta_{c}=20 \mathrm{deg}$., the values of $L_{c}$ were $0.22 \mathrm{~mm}$ for $\theta_{s}$ with 5 deg., $0.24 \mathrm{~mm}$ for $\theta_{s}$ with 10 deg., and $0.26 \mathrm{~mm}$ for $\theta_{s}$ with 20 deg. From the numerical values, the differences in each condition were small. What should be careful here is that these difference would vary according to a pre-determined condition of $h_{c}$.

The influence of tool orientation on $L_{f}$ is displayed in Figure 11. In the figure, each curve for respective $\theta_{s}$ in graph legends represented decreasing tendency with increasing $\theta_{c}$ in any case. Unlike the decreasing tendency of $L_{c}$, that of $L_{f}$ mildly weakened with increasing $\theta_{s}$. The differences between each curve became larger with increasing $\theta_{c}$, whereas it can be presumed that there existed the limit of $L_{f}$ in the case of $\theta$ having an angle more than $20 \mathrm{deg}$. The results revealed that the changing tendency of $L_{f}$ was completely different from that of $L_{c}$. In contrast, it was distinctive that $L_{f}$ became completely equal in any case without $\theta_{c}$. In other words, the fact means that these intersection condition between a torus section and $h_{f}$ pseudo plane coincided perfectly despite the difference in $\theta_{s}$.

The influence of tool orientation on $s l_{f d}$ is shown in Figure 12. In the figure, each curve for respective $\theta_{s}$ in graph legends represented decreasing tendency with increasing $\theta_{c}$ in any case. They can be seemingly observed as straight decline. Moreover, the differences between each curve became gradually smaller with increasing $\theta_{c}$. The results denoted that the values of $s l_{f d}$ appeared to converge towards a certain value with increasing $\theta_{c}$. As an example of numerical results, when $\theta_{c}=20$ deg., the values of $s l_{f d}$ were $4.82 \mathrm{~mm}$ for $\theta_{s}$ with 5 deg., $4.71 \mathrm{~mm}$ for $\theta_{s}$ with 10 deg., and $4.32 \mathrm{~mm}$ for $\theta_{s}$ with $20 \mathrm{deg}$. Numerically, the values of $s l_{f d}$ are likely to become convergent in the case of $\theta_{c}$ with more than $20 \mathrm{deg}$.

The influence of tool orientation on $s l_{c a l}$ is given in Figure 13. In the figure, each curve for respective $\theta_{s}$ in graph legends represented decreasing tendency with increasing $\theta_{c}$ in any case. The differences between each curve became larger with increasing $\theta_{c}$, whereas it could be presumed that there existed the limit of $s l_{c a l}$ in the case of $\theta_{c}$ having an angle more than $20 \mathrm{deg}$. The results indicated that contact arc length was largely unaltered in any case. They also implied that the intersection conditions between a torus section and workpiece's surface were almost identical despite the difference in $\theta$. In the pre-determined condition of $d_{d o c}$, the values of $s l_{c a l}$ were $0.95 \mathrm{~mm}$ in any case when $\theta_{c}=0$ deg. In addition, when $\theta_{c}=20$ deg., they were $0.91 \mathrm{~mm}$ for $\theta_{s}$ with 5 deg., $0.92 \mathrm{~mm}$ for $\theta_{s}$ with $10 \mathrm{deg}$., and $0.93 \mathrm{~mm}$ for $\theta_{s}$ with $20 \mathrm{deg}$. From the numerical values, there was little difference in each tool orientation. 


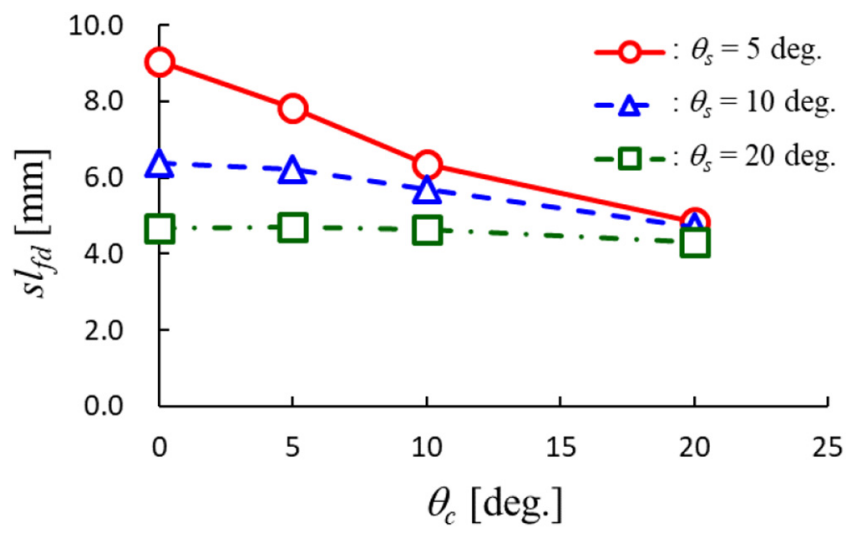

Fig. 12. Influence of $\theta_{s}$ and $\theta_{c}$ on $s l_{f d}$.

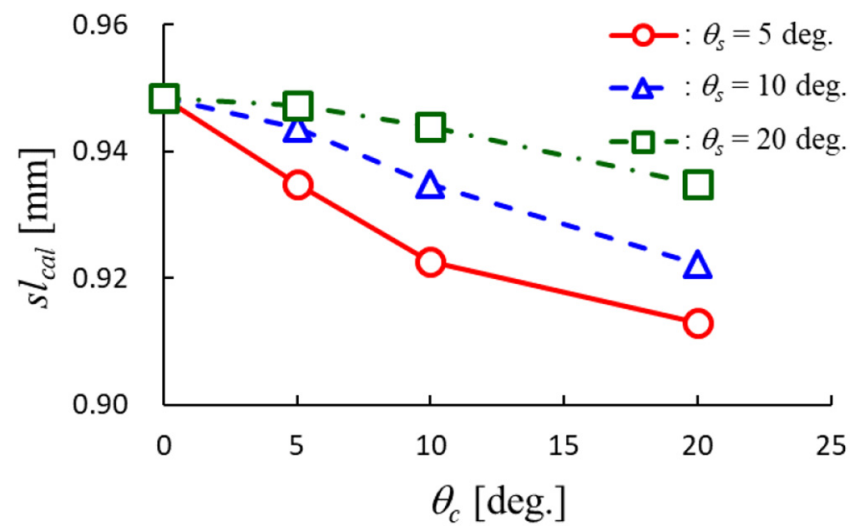

Fig. 13. Influence of $\theta_{s}$ and $\theta_{c}$ on $L_{c a l}$.

The influence of tool orientation on $v_{c s}$ is represented in Figure 14. In the figure, each curve for respective $\theta_{s}$ in graph legends showed increasing tendency with increasing $\theta_{c}$ in any case. The increasing tendency gradually diminished with increasing $\theta_{s}$. Moreover, the differences between each curve became smaller with increasing $\theta_{c}$. As a numerical example, when $\theta_{s}=20 \mathrm{deg}$. and $\theta_{c}=5 \mathrm{deg}$., the value of $v_{c s}$ was $47.92 \mathrm{~m} / \mathrm{min}$. Likewise, when $\theta_{s}=5 \mathrm{deg}$. and $\theta_{c}=20$ deg., the value of $v_{c s}$ was $47.92 \mathrm{~m} / \mathrm{min}$. In the two machining states, $\theta$ was $20.52 \mathrm{deg}$. It was obvious from the results that the value of $v_{c s}$ was completely identical under the same $\theta$.

The influence of tool orientation on $t_{\text {ave }}$ is displayed in Figure 15. In the figure, each curve for respective $\theta_{s}$ in graph legends indicated decreasing tendency with increasing $\theta_{c}$ in any case. The differences between each curve became larger with increasing $\theta_{c}$, whereas it can be presumed that there existed the limit of $t_{\text {ave }}$ in the case of $\theta_{c}$ having an angle more than $20 \mathrm{deg}$. The results implied that an instantaneous cutting force acting on a cutting edge decreased with increasing $\theta$. With reference to the variation of $t_{\text {ave }}$, the decreasing rate would be especially prominent in the small value of $\theta_{s}$.

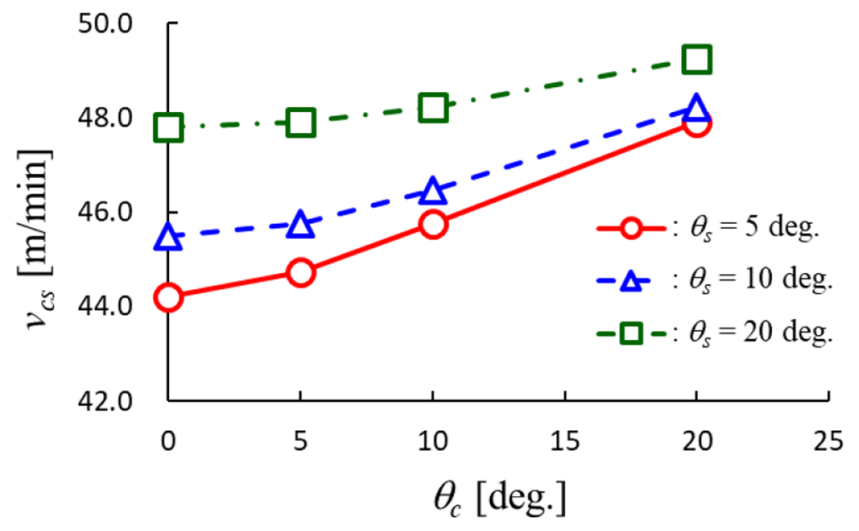

Fig. 14. Influence of $\theta_{s}$ and $\theta_{c}$ on $v_{c s}$.

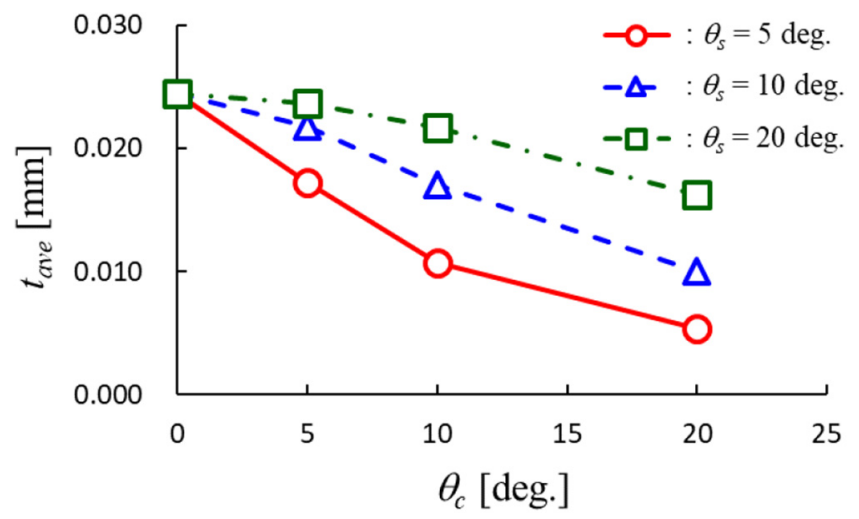

Fig. 15. Influence of $\theta_{s}$ and $\theta_{c}$ on $t_{\text {ave }}$.

Wojciechowski et al. reported several relationships between three average forces in cutting process and average uncut chip thickness under some inclination angles in ball end milling [37]. From their results, tangential and radial average forces increased with increasing average uncut chip thickness, whereas axial one decreased in the same condition. In addition, the changing tendencies of three average forces became moderate with increasing an inclination angle. They also provided that there were precipitous variations of average forces within an inclination angle less than $15 \mathrm{deg}$. In contrast, the variations had a little change when an inclination angle was more than 15 deg. It was also denoted that a width of flank wear depended on an amount of average forces.

Budak and Ozlu investigated some relationships between cutting forces and feed rates in machining process [38]. The results showed that a cutting force increased with increasing a feed rate. In addition, an amount of cutting force depended on a depth of cut.

Bouzakis et al. studied some relationships between tool orientation and surface roughness in ball end milling [39]. Their results revealed that oblique plunge up and down milling were extremely desirable tool orientations in terms of surface roughness and cutting force. Especially, oblique 
plunge up milling was recommended from the experimental results obtained using some materials. Moreover, surface roughness around $10 \mathrm{deg}$. could stay as the smallest value.

As one possible conclusion from the results and findings described above, optimal angle of $\theta$ is in a range of $15-20 \mathrm{deg}$. in filleted end milling without $\theta_{c}$. This condition can achieve both high production efficiency and decent surface feature since two path intervals $L_{c}$ and $L_{f}$ are theoretically large. Oblique plunge up milling is also recommended highly under the condition of a tool inclination along a tool feed direction.

\section{Conclusions}

In this study, theoretical approaches were demonstrated to determine machining conditions affecting machined surface topography in filleted end milling. After geometrical description was explained to model multi-axis filleted end milling, multi-layer approach and the other theoretical approaches were proposed to obtain decent surface topography generated in filleted end milling. The analytical example focusing on tool orientation was given with discussion. As a result, some characteristics of theoretical approaches were revealed with visual evidences. The findings led to one possible conclusion that optimal angle of $\theta$ was in a range of $15-20 \mathrm{deg}$. without $\theta_{c}$. Oblique plunge up milling is also recommended highly under the condition of a tool inclination along a tool feed direction.

As a future work, the further detailed analysis will be conducted in a wide variety of conditions, and the experimental verification will be made to evaluate validity and applicability of theoretical approaches proposed in this study.

\section{Nomenclature}

$\mathbf{X}, \mathbf{Y}, \mathbf{Z}$
$\mathbf{M X}, \mathbf{M Y}, \mathbf{M Z}$
$\mathbf{T X}, \mathbf{T Y}, \mathbf{T Z}$
$\theta$
$\theta_{s}$
$\theta_{c}$
$R$
$R$
$R_{b}$
$\mathbf{P}_{\mathrm{tc}}$
$\mathbf{P}_{\mathbf{e}}$
$\mathbf{T P}_{\mathbf{e}}$
$\mathbf{R}_{\mathbf{T}}$
$\psi$
$\xi$
$\zeta$

global, stationary coordinate system (O coordinate system)

$\mathrm{M}$ coordinate system

$\mathrm{T}$ coordinate system

tool inclination angle [rad]

tool inclination angle in feed direction [rad]

tool inclination angle in cross-feed direction

tool radius

corner radius of cutting edge

major radius of torus

tool center

a cutting point on a cutting edge

a cutting point on a cutting edge in $\mathrm{T}$ coordinate system

transformation matrix

positional angle in a filleted part on a cutting edge [rad]

initial angle between TY and each cutting edge [rad]

rotational angle of a cutting edge [rad]
$n_{c e}$
$L_{c}$
$L_{f}$
$h_{c}$
$h_{f}$
$\mathbf{P}_{\text {base }}, \mathbf{P}_{\text {start }}, \mathbf{P}_{\text {end }}$
$\mathbf{P}_{\text {ap }}, \mathbf{P}_{\text {scp }}, \mathbf{P}_{\mathbf{h p}}$
$\mathbf{t}_{\text {base }}, \mathbf{t}_{\mathrm{ap}}, \mathbf{t}_{\mathrm{scp}}$
$\mathrm{n}_{\mathrm{s}}$
$\mathrm{m}_{\mathrm{scp}}, \mathrm{u}_{\mathrm{scp}}$
$\gamma$
the number of cutting edges
path interval in cross-feed direction [mm]
path interval in feed direction [mm] scallop height [mm]
feed mark's height [mm]
a positional vector
in each applicable part
a tangent vector in each applicable part
surface normal
a directional vector in each applica- ble part
an angular parameter for determin- ing an arbitrary position on a circle with $R_{b}$ [rad]
$\eta$
a direction cosine between $\mathbf{t}_{\mathbf{a p}}$ and an applicable surface or plane
$d_{s} \quad$ a distance between $\mathbf{P}_{\text {ap }}$ and $\mathbf{P}_{\mathbf{h p}}$ [mm]
$f_{t} \quad$ feed per tooth $[\mathrm{mm} /$ tooth $]$
$\omega \quad$ the rotational angle between $\mathbf{X}$ axis
and TX - TZ plane [rad]
$S l_{f d} \quad$ maximum frictional distance $[\mathrm{mm}]$
$S l_{c a l} \quad$ maximum contact arc length [mm]
$v_{c s} \quad$ maximum cutting speed $[\mathrm{m} / \mathrm{min}]$
$t_{u c t} \quad$ uncut chip thickness [mm]
$t_{\text {ave }} \quad$ average uncut chip thickness [mm]
$\alpha_{f d}, \beta_{f d}, \alpha_{c a l}, \alpha_{h f}$
an angular parameter
$\alpha_{c s}, \alpha_{u c t}, \beta_{u c t}$

The authors would like to thank the financial support provided by OSG Fund, Shotoku Science Foundation, and the research grant from Faculty of Science and Technology, Seikei University.

\section{References}

1. P.G. Benardos, G.C. Vosniakos, Predicting surface roughness in machining: a review, Int. J. Mach. Tools Manuf. 43, 833-844 (2003)

2. C. Lu, Study on prediction of surface quality in machining process, J. Mater. Process. Technol. 205, 439-450 (2008)

3. S.G. Croll, Surface roughness profile and its effect on coating adhesion and corrosion protection: a review, Prog. Org. Coat. 148, 105847 (2020)

4. S.J. Zhang, S. To, S.J. Wang, Z.W. Zhu, A review of surface roughness generation in ultra-precision machining, Int. J. Mach, Tools Manuf. 91, 76-95 (2015)

5. H.L. Fisher, J.T. Elrod, Surface finish as a function of tool geometry and feed - a theoretical approach, Microtechnic 25, 175-178 (1971)

6. W.A. Kline, R.E. DeVor, I.A. Shareef, The prediction of surface accuracy in end milling, ASME. J. Eng. Ind. 104, 272-278 (1982)

7. K-H. Fuh, C-F. Wu, A proposed statistical model for surface quality prediction in end milling of $\mathrm{Al}$ alloy, Int. J. Mach Tools Manuf. 35, 1187-1200 (1995)

8. H. Paris, G. Peigne, R. Mayer, Surface shape prediction in high speed milling, Int. J. Mach, Tools Manuf. 44, 1567-1576 (2004) 
9. Y. Mizugaki, K. Kikkawa, H. Terai, M. Hao, T. Sata, Theoretical estimation of machined surface profile based on cutting edge movement and tool orientation in ball-nosed end milling, CIRP Annals. 52, 49-52 (2003)

10. T. Sekine, T. Obikawa, M. Hoshino, Establishing a novel model for 5-axis milling with filleted end mill, J. Adv. Mech. Des. Syst. Manuf. 6, 296-309 (2012)

11. T. Sekine, T. Obikawa, Novel path interval determination in 5-axis flat end milling. Appl. Math. Model. 39, 3459-3480 (2015)

12. A.M. Khorasani, M.R.S. Yazdi, M.S. Safizadeh, Analysis of machining parameters effects on surface roughness: a review, Int. J. Comput. Mater. Sci. Surf. Eng. 5, 68-84 (2012)

13. T. Matsumura, S. Takahashi, Micro dimple milling on cylinder surfaces, J. Manuf. Process. 14, 135-140 (2012)

14. I. Perez, A. Madariaga, P.J. Arrazola, M. Cuesta, D. Soriano, An analytical approach to calculate stress concentration factors of machined surfaces, Int. J. Mech. Sci. 190, 106040 (2021)

15. Y. Quinsat, L. Sabourin, C. Lartigue, Surface topography in ball end milling process: description of a 3D surface roughness parameter, J. Mater. Process. Technol. 195, 135-143 (2008)

16. R.B. Käsemodel, A.F. de Souza, R. Voigt, I. Basso, A.R. Rodrigues, CAD/CAM interfaced algorithm reduces cutting force, roughness, and machining time in free-form milling. Int. J. Adv. Manuf. Technol. 107, 1883-1900 (2020)

17. Y.K. Choi, A. Banerjee, J.W. Lee, Tool path generation for free form surfaces using Bézier curves/surfaces, Comput. Ind. Eng. 52, 486-501 (2007)

18. T. Obikawa, T. Sekine. A higher-order formula of path interval for tool-path generation, Int. J. Autom. Technol. 5, 663-668 (2011)

19. L.T. Tunc, Smart tool path generation for 5-axis ball-end milling of sculptured surfaces using process models, Robot. Comput. Integr. Manufactur. 56, 212-221 (2019)

20. G.M. Mladenovic, L.M. Tanovic, K.F. Ehmann, Tool path generation for milling of free form surfaces with feedrate scheduling, FME Trans. 43, 9-15 (2015)

21. T. Sekine, T. Obikawa, Novel path interval determination in 5-axis flat end milling, Appl. Math. Model. 39, 3459-3480 (2015)

22. D. Plakhotnik, B. Lauwers, Computing of the actual shape of removed material for five-axis flat-end milling, Comput. Aided Des. 44, 1103-1114 (2012)

23. S. Segonds, P. Seitier, C. Bordreuil, F. Bugarin, W. Rubio, J. M. Redonnet, An analytical model taking feed rate effect into consideration for scallop height calculation in milling with torus-end cutter, J. Intell. Manuf., 30, 1881-1893 (2019)

24. T. Sekine, T. Obikawa, M. Hoshino, Establishing a novel model for 5-axis milling with filleted end mill, J. Adv. Mech. Des. Syst. Manufactur. 6, 296-309 (2012)
25. T. Sekine, A 3D geometrical consideration of path interval in filleted end milling, J. Jpn. Soc. Abras. Technol. 60, 515-519 (2016) (in Japanese)

26. T. Sekine, A computational algorithm for path interval determination in multi-axis filleted end milling, Adv. Sci. Technol. Res. J. 14, 198-205 (2020)

27. R.A. Mali, T.V.K. Gupta, J. Ramkumar, A comprehensive review of free-form surface milling- Advances over a decade, J. Manufactur. Process. 62, 132-167 (2021).

28. I. Mukherjee, P.K. Ray, A review of optimization techniques in metal cutting processes, Comput. Ind. Eng. 50, 15-34 (2006)

29. A.M. Khorasani, M.R.S. Yazdi, M.S. Safizadeh, Analysis of machining parameters effects on surface roughness: a review, Int. J. Comput. Mater. Sci. Surf. Eng. 5, 68-84 (2012).

30. I. Perez, A. Madariaga, P.J. Arrazola, M. Cuesta, D. Soriano, An analytical approach to calculate stress concentration factors of machined surfaces, Int. J. Mech. Sci. 190, 106040 (2021)

31. R.B. Käsemodel, A.F. de Souza, R. Voigt, I. Basso, A.R. Rodrigues, CAD/CAM interfaced algorithm reduces cutting force, roughness, and machining time in free-form milling, Int. J. Adv. Manuf. Technol. 107, 1883-1900 (2020)

32. I. Lazoglu, S.E.L. Khavidaki, A. Mamedov, H. Erdim, Process optimization via feedrate scheduling in milling. In: The International Academy for Production Engineering, edited by L. Laperrière, G. Reinhart, CIRP Encyclopedia of Production Engineering. Springer, Berlin, Heidelberg (2014)

33. M. Habibi, Z.M. Kilic, Y. Altintas, Minimizing flute engagement to adjust tool orientation for reducing surface errors in five-axis ball end milling operations, ASME. J. Manuf. Sci. Eng. 143, 021009 (2021)

34. X. Zhang, J. Zhang, X. Zheng, B. Pang, W. Zhao, Tool orientation optimization of 5 -axis ball-end milling based on an accurate cutter/workpiece engagement model, CIRP J. Manufactur. Sci. Technol. 19, 106-116 (2017)

35. S. Lotfi, B. Rami, B. Maher, D. Gilles, B. Wassila, An approach to modeling the chip thickness and cutter workpiece engagement region in 3 and 5 axis ball end milling, J. Manuf. Process. 34, 7-17 (2018)

36. N. Masmiati, A.A.D. Sarhan, Optimizing cutting parameters in inclined end milling for minimum surface residual stress Taguchi approach, Measurement 60, 267-275 (2015)

37. S. Wojciechowski, R.W. Maruda, P. Nieslony, G.M. Krolczyk, Investigation on the edge forces in ball end milling of inclined surfaces, Int. J. Mech. Sci. 119, 360-369 (2016)

38. E. Budak, E. Ozlu, Development of a thermomechanical cutting process model for machining process simulations, CIRP Ann. 57, 97-100 (2008)

39. K.D. Bouzakis, P. Aichouh, K. Efstathiou, Determination of the chip geometry, cutting force and roughness in free form surfaces finishing milling, with ball end tools, Int. J. Mach. Tools Manufact. 43, 499-514 (2003)

Cite this article as: Tsutomu Sekine, Theoretical approaches for determining machining conditions affecting a machined surface topography in filleted end milling, Int. J. Simul. Multidisci. Des. Optim. 12, 27 (2021) 